\title{
An Evaluation of Treatment Outcomes in a Cohort of Clients on the DOTS Strategy, 2012-2016
}

\author{
Ato Kwamena Tetteh $\left(\mathbb{D},{ }^{1}\right.$ Edward Agyarko, ${ }^{2}$ Joseph Otchere, ${ }^{3}$ \\ Langbong Bimi, ${ }^{4}$ and Irene $\mathrm{Ayi}^{3}$ \\ ${ }^{1}$ Laboratory Department, Metropolitan Hospital, P.O. Box 174, Cape Coast, Ghana \\ ${ }^{2}$ Department of Community Medicine and Health, Anglican University College of Technology, P.O. Box 74, Nkoranza, Ghana \\ ${ }^{3}$ Noguchi Memorial Institute for Medical Research, Department of Parasitology, College of Health Sciences, University of Ghana, \\ Legon, Accra, Ghana \\ ${ }^{4}$ Department of Animal Biology and Conservation Sciences, University of Ghana, Legon, Accra, Ghana
}

Correspondence should be addressed to Ato Kwamena Tetteh; aktetteh@outlook.com

Received 16 October 2017; Accepted 18 January 2018; Published 15 February 2018

Academic Editor: William N. Rom

Copyright (C) 2018 Ato Kwamena Tetteh et al. This is an open access article distributed under the Creative Commons Attribution License, which permits unrestricted use, distribution, and reproduction in any medium, provided the original work is properly cited.

\begin{abstract}
We present, for the first time, an evaluation of treatment outcomes in a cohort at a TB referral centre in the Central Region of Ghana. Of the 213 clients placed on DOTS, 59.2\% (126/213) were sputum smear-positive. An overall cure rate of $90.2 \%(51.6 \%$ cured $+37.6 \%$ completed) and a death rate of $8.5 \%$ (18/213) were estimated. Of the number of clients who died, 5.7\% (12/213) were males $\left(\chi^{2}=2.891, p=0.699 ; \mathrm{LR}=3.004, p=0.699\right)$. Deaths were only recorded among clients who were $>19$ years old $\left(\chi^{2}=40.319\right.$, $p=0.099 ; \mathrm{LR}=41.244, p=0.083)$. Also, $0.9 \%(2 / 213)$ was lost to follow-up, while $1.4 \%(3 / 213)$ had treatment failure. In total, $13.6 \%$ (7.0\%, 15/213 males, and 6.6\%, 14/213 females) of clients who were placed on DOTS were HIV seropositive. Ages of 40-49 years had the highest number, 13/213 (6.1\%), infected with HIV, though the difference among the remaining age groups was not statistically significant $\left(\chi^{2}=9.621, p=0.142\right)$. Furthermore, $7.0 \%(15 / 213)$ had TB/HIV coinfection. Out of them, 9 were cured and 5 died at home, while 1 had treatment failure. Tuberculosis/HIV infection prevention advocacy and interventions that address sociodemographic determinants of unfavourable treatment outcomes are urgently required to augment national efforts towards control.
\end{abstract}

\section{Introduction}

Successful tuberculosis (TB) treatment has a positive significant effect on the control of TB. Therefore, completing prescribed medication in active cases is of prime importance to TB control programmes [1]. Globally, the Directly Observed Therapy Short-course (DOTS) has been accepted as a strategy to the cure of tuberculosis [2]. The DOTS strategy has been used in Ghana since 1994, and it remains the medication strategy of choice [3]. This strategy aims at improving patients' commitment to medication and therefore minimizes development of drug resistance to contemporary remedies [4]. With this strategy, it is expected that 80 to $90 \%$ of patients will undergo smear conversion within two to three months of treatment [5].
The change in bacterial infection status of sputum of patients from initial Acid-Fast Bacilli (AFB) positive to negative after treatment is referred to as sputum smear conversion (SSC). Smear conversion rate (SCR) at two months of intensive phase (IP) and at three months of IP (extended IP) is a significant operational indicator, as it shows the capacity of the programme to maintain the patients on treatment. It also provides an objective verification for the client's response to therapy and consequently the treatment outcome [5].

The Global TB Report (2011) estimated 86 smear-positive pulmonary TB cases per 100,000 people per year [6]. It has been established that one untreated infectious tuberculosis patient is likely to infect 10 to 15 persons annually [7]. Also, a number of factors have been acknowledged as delaying the time to smear and culture conversion. These include 


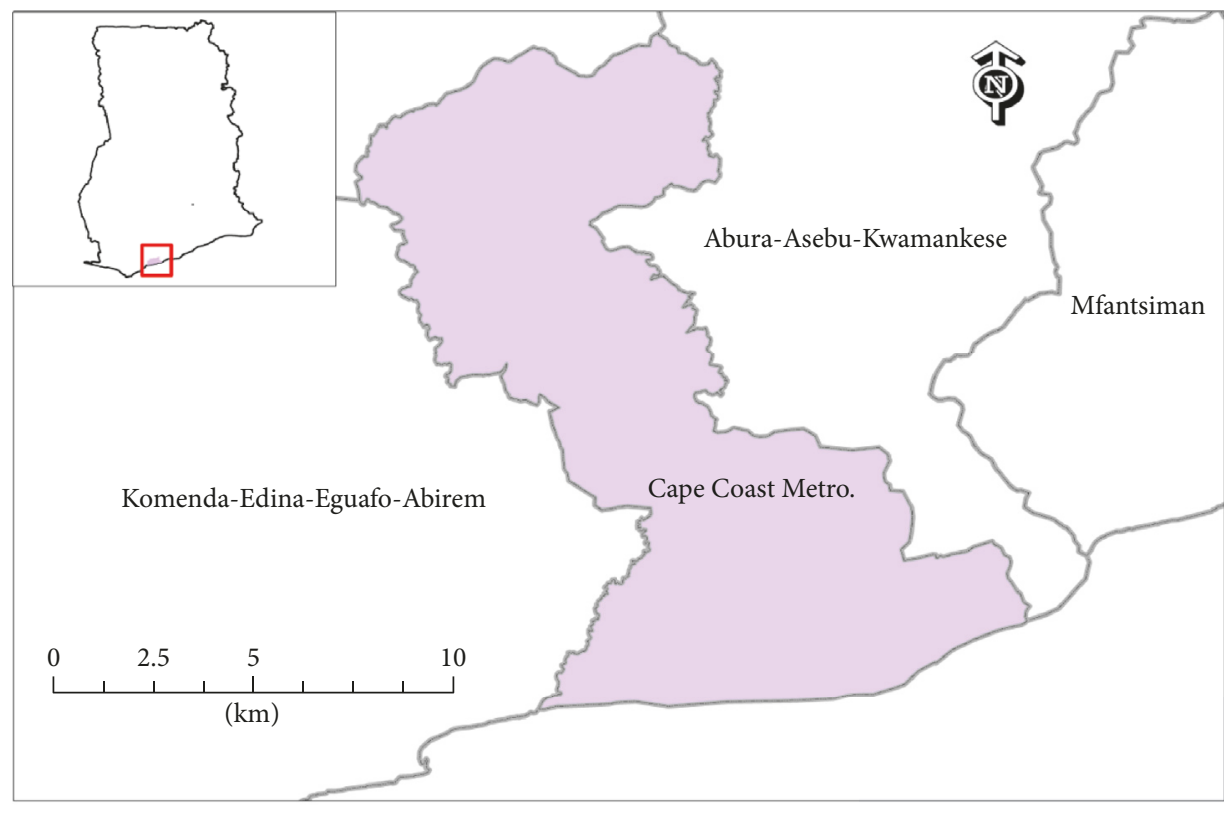

Cape Coast Metro.

District boundary

Figure 1: Map of Cape Coast Metropolitan Area (credit: Dr. Charles Gyamfi, Department of Civil Engineering, Tshwane University of Technology, Pretoria, South Africa).

high intensity initial sputum smear AFB grade, cavitary lesion, uncontrolled hyperglycaemia/diabetes mellitus, old age, certain ethnic population (e.g., Hispanic), multidrugresistant tuberculosis, initial treatment with less than four antitubercular drugs, and non-rifampicin-based treatment regimens [8-14].

Generally, only sputum culture confirms noncontagious clients. However, lack of culture facilities in most TB centres, except for Regional/Teaching Hospitals in Ghana, necessitates the use of serial negative smears (SNS) before removal of infection control measures. Sputum examination has been described worldwide as the most definite, cost-effective, and dependable test for the diagnosis of pulmonary TB. It confirms the diagnosis and provides information on whether there is positive and progressive response to treatment. At the end of treatment, it shows if clients have been cured. The Metropolitan Hospital (MH), located within the Cape Coast Metropolitan Area (CCMA), has a TB testing centre and it is the only TB referral centre in the Central Region of Ghana. Yearly, about 250-400 new diagnostic cases are referred for testing from within CCMA and adjoining communities. A third of these return test results to treatment centres within their communities for management. The remaining, who reported to $\mathrm{MH}$ for consultation, may be recruited onto the DOTS strategy, which is comprehensively elaborated in the Tuberculosis Case Management Desk Aide. Over the past decades, no on-site assessment has been done regarding the conversion rates and performance of the current DOTS strategy. This study presents the outcome of a five-year retrospective evaluation of TB-confirmed and TB-suggestive clients placed on the DOTS strategy.

\section{Materials and Methods}

2.1. Setting. The entire Cape Coast Metropolis covers an area of $122 \mathrm{~km}^{2}$ and it is located on longitude $1^{\circ} 15^{\prime} \mathrm{W}$ and latitude $5^{\circ} 06^{\prime} \mathrm{N}$ (Figure 1). The Metropolitan Hospital, Cape Coast, was established in 1939 and it is located on the Beulah Road (Beach Road) between the Nursing and Midwifery Training College and St. Augustine's College. The hospital serves the entire Metropolis and it is the first referral facility for all Government Health Centres and Clinics in the area, as well as the adjoining communities (https://www.google.com .gh/maps/@5.1038147,-1.2596938,587m/data=!3m1!1e3). The hospital has a separate special structure, christened "Chest Ward," where all clients diagnosed or suspected of having TB are admitted.

2.2. Study Design and Population. This was a five-year retrospective study comprising all diagnosed and suspected clients who were admitted and enrolled on the DOTS strategy from 1 January 2012 to 31 December 2016 (Table 1). In total, 213 male and female clients of all ages were included in the study. Compiled data included clients who were acid fast bacilli(AFB-) positive or negative. All clients had persistent cough, with/without expectoration, increased axillary temperature, loss of appetite, and weight loss.

2.3. Data Collection. Secondary data were compiled from TB patients' registers at the Chest Ward. Client information collected included age, sex, form of tuberculosis (pulmonary or extrapulmonary), type of tuberculosis (smear-positive or smear-negative), category of tuberculosis (new cases 
TABLE 1: Recommended DOTS treatment regimen for each category of tuberculosis in Ghana.

\begin{tabular}{|c|c|c|c|}
\hline \multirow{2}{*}{$\begin{array}{l}\text { Patient } \\
\text { category }\end{array}$} & \multirow[t]{2}{*}{ Definition } & Initial phase treatment ${ }^{1 *}$ & $\begin{array}{l}\text { Continuation phase } \\
\text { treatment }\end{array}$ \\
\hline & & Daily (28 doses/month) & Daily (28 doses/month) \\
\hline \multirow{6}{*}{ I } & All new cases & \multirow{5}{*}{$\begin{array}{c}2(H R Z E)^{2}=56 \text { doses of } \\
\text { HRZE }\end{array}$} & \multirow{5}{*}{$4(H R)=112$ doses of HR } \\
\hline & (i) New smear-positive & & \\
\hline & (ii) New smear-negative PTB & & \\
\hline & (iii) Concomitant HIV disease & & \\
\hline & (iv) Extrapulmonary TB & & \\
\hline & $\begin{array}{l}\text { Previously treated sputum } \\
\text { smear-positive PTB }\end{array}$ & & \multirow{4}{*}{$5(H R E)=140$ doses of HRE } \\
\hline \multirow{3}{*}{ II } & (i) Relapse & $2(H R Z E) S+1(H R Z E)=84$ & \\
\hline & (ii) Treatment after interruption & doses of HRZE + 56 doses & \\
\hline & (iii) Treatment failure & of $\mathrm{S}$ & \\
\hline $\mathrm{III}^{3}$ & Children below 12 years & $2(H R Z)=56$ doses of $\mathrm{HRZ}$ & $4(H R)=112$ doses of $\mathrm{HR}$ \\
\hline \multicolumn{4}{|c|}{$\begin{array}{l}\text { Table } 1 \text { is adopted from the National Tuberculosis Programme (NTP) Training Manual, } 2012 .{ }^{1} \text { Direct observation of treatment intake is required and always in } \\
\text { regimens including rifampicin. }{ }^{2} \text { Streptomycin may be used instead of ethambutol. In meningitis, ethambutol should be replaced by streptomycin. }{ }^{3} \text { In children with } \\
\text { meningitis, add streptomycin in the initial phase. Category I: new clients; category II: previously treated clients; category III: children }<12 \text { years. TB drugs and } \\
\text { codes: H: isoniazid; R: rifampicin; Z: pyrazinamide; S: streptomycin; E: ethambutol. Codes for Fixed Drug Combinations (FDC): (HR): isoniazid + rifampicin; } \\
\text { (HRZ): isoniazid + rifampicin + pyrazinamide; (HRZ): isoniazid + rifampicin + pyrazinamide + ethambutol. * The administration of these drugs has been } \\
\text { expansively explained in the } 2012 \text { edition of the NTP Training Manual. }\end{array}$} \\
\hline
\end{tabular}

or relapse or retreatment cases), and treatment outcome. Treatment outcome of clients was evaluated in harmony with World Health Organization reference and classified as cured, completed (treatment completed), default, treatment failure, death, and others. In this study, treatment was based on pretreatment sputum smear grading (PSSG) and/or TBsuggestive symptoms/sign, coupled with $\mathrm{X}$-ray suggestive images. In addition, the HIV statuses of $\mathrm{TB}$ clients were confirmed from their medical records. Those whose status was not already known were tested using the preliminary First Response $\mathrm{HIV}^{\circledR} 1 / 2$ and the confirmatory OraQuick ${ }^{\circledR} \mathrm{HIV}$ $1 / 2$.

\subsection{Treatment Regimen. See Table 1.}

2.5. Ethical Approval. Permission was sought from the Metropolitan Hospital and the Chest Centre to access TB clients' archived data. Path numbers of patients were not recorded at all in order to conceal their identity. It was emphasized that data presented in this study will strictly be for academic purposes only to help improve management of patients in the future and will cause no harm in accordance with the Declaration of Helsinki (1964).

2.6. WHO Operational Definitions. New smear-positive TB case is a patient who has never had treatment for TB or who has taken anti-TB drugs for less than one month. Treatment outcome was divided into six categories according to the National TB Control Programme guidelines: cured: a patient who was smear-positive at diagnosis and was smear-negative at the end of the last month of treatment and on at least one previous occasion; treatment completed: any patient who was smear-negative but with suggestive signs/symptoms of $\mathrm{TB}$ at the time of diagnosis and had completed treatment, with or without sputum smear results; died: any patient who died irrespective of the cause of death during the course of treatment; treatment failure: any patient who remained or became positive at the end of the fifth month or later during treatment; treatment defaulter: any patient who has interrupted treatment two consecutive months or more after the date of the last attendance during the course of treatment; transfer-out; a patient who was transferred to another treatment centre and whose treatment outcome is unknown.

2.7. Data Analysis. All records from TB patients' registers from 2012 to 2016 were recoded, concealing the identities of clients. Data were double-entered into Microsoft Excel ${ }^{\circledR}$ and then cleaned (checked and edited) of inconsistencies. Completeness of the TB patients' registers after cleaning was approximately 99.5\%. Data were later transported into SPSS (version 20) for analysis. Results were expressed as absolute frequencies $(n)$ and percentages (\%). Proportions with $95 \%$ confidence intervals and Chi-square test were employed to compare different groups and $p$ value less than 0.05 was considered statistically significant. Smear conversion was estimated as the time (in weeks) from initiation of treatment to first of the two serial negative smears for AFB. Cure rate was deduced according to the WHO cure rate/treatment success rate definition as follows: the proportion of new smear-positive TB cases registered under DOTS in a given year that successfully completed treatment, whether with ("cured") or without ("treatment completed") bacteriologic evidence of success. The study consolidated the respective yearly data to obtain overall rates for the period under review.

\section{Results}

3.1. Sociodemographic Characteristics. In total, 213 clients whose data were available in tuberculosis (TB) treatment registers from 2012 to $2016(2012=44 ; 2013=55 ; 2014=48$; 
TABLE 2: Age and sex distribution of clients from 2012 to 2016.

\begin{tabular}{lccc}
\hline \multirow{2}{*}{ Age group (years) } & \multicolumn{2}{c}{ Sex of clients* } & \multirow{2}{*}{ Total $^{* *}$} \\
& Female (\%) & Male (\%) & \\
\hline$<10$ & $2(0.9)$ & - & 2 \\
$10-19$ & $5(2.3)$ & $6(2.8)$ & 11 \\
$20-29$ & $12(5.6)$ & $23(10.8)$ & 35 \\
$30-39$ & $14(6.6)$ & $30(14.1)$ & 44 \\
$40-49$ & $9(4.2)$ & $42(19.7)$ & 51 \\
$50-59$ & $9(4.2)$ & $31(14.6)$ & 40 \\
$\geq 60$ & $3(1.4)$ & $27(12.7)$ & 30 \\
\hline Total & $54(25.4)$ & $159(74.6)$ & 213 \\
\hline
\end{tabular}

${ }^{*}$ Significantly more males than females were recruited for treatment $\left(\chi^{2}=\right.$ $16.9, p=0.013) .{ }^{* *}$ Total number of clients on the Directly Observed Treatment Short-course (DOTS).

$2015=44 ; 2016=22)$ were included in the analysis. These consisted of 54 females and 159 males $\left(\chi^{2}=16.9, p=0.013\right)$, aged between 4 and 79 years (mean age $=42.37 \pm 15.33$ ) (Table 2). Of the total, 38.0\% were from Cape Coast, while $62.0 \%$ were from adjoining rural communities.

3.2. Pretreatment Sputum Smear Grade and Treatment Outcome among Clients. Of the total number of clients on DOTS, $40.8 \%(87 / 213)$ were sputum smear-negative (SSN), while the remaining $59.2 \%(126 / 213)$ were sputum smear-positive (SSP) (Table 3 ). In all, 173 were placed in category I, 38 in category II, and 2 in category III (table not shown). Percentage distribution of the pretreatment sputum smear grade (PSSG) among clients is as indicated in Table 4. Out of the total SSP clients, $77.0 \%$ (97/126) were males and $23 \%$ $(29 / 126)$ were females. The overall cure rate, $90.2 \%$, was determined by adding the percentage cured to the percentage that completed the treatment.

3.3. Death Rate for TB Clients from 2012 to 2016. According to the records, two clients were lost to follow-up (Table 3). Of these, one was SSN, while the other was SSP. In total, $8.5 \%(18 / 213)$ of clients on DOTS died (17 at home and 1 at the Chest Ward) during the period under review. The annual death distribution over the entire period is as follows: 2012, $0.5 \%$ (1/213); 2013, 1.9\% (4/213); 2014, 2.8\% (6/213); 2015, 2.4\% $(5 / 213)$; and 2016, $0.9 \%$ (2/213). Among the sexes, six females $(2.8 \%)$ died compared to 12 males $(5.7 \%)\left(\chi^{2}=2.891, p=\right.$ 0.699 ; likelihood ratio $=3.004, p=0.699)$. With regard to the age groups, no death was recorded among clients $\leq 19$ years; in the age group of 20-29 years, one death was recorded; in the age group of 30-39 years, three deaths were recorded; in the age group of 40-49 years, six deaths were recorded; in the age group of 50-59 years, six deaths were recorded; and in the age group of $\geq 60$ years, one death was recorded $\left(\chi^{2}=40.319\right.$, $p=0.099$; likelihood ratio $=41.244, p=0.083$ ).

3.4. HIV-Infected TB Clients on DOTS from 2012 to 2016. In total, $13.6 \%$ (29/213) who were HIV seropositive were placed on DOTS (Table 4). This was based on either a positive PSSG or a TB-suggestive sign/symptom, coupled with an X-ray

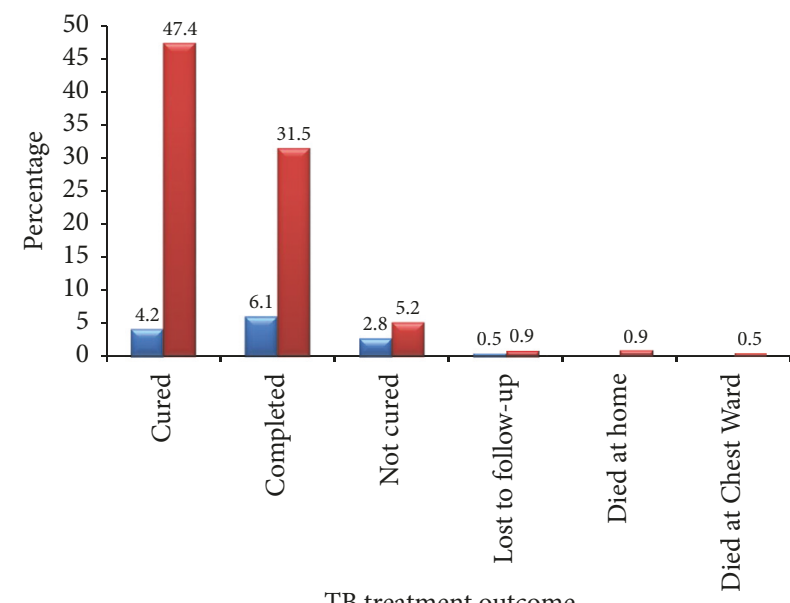

TB treatment outcome

$\square$ HIV seropositive
$\square$ HIV seronegative

FIGURE 2: Treatment outcomes for TB/HIV coinfected clients.

image. Although more males were placed on DOTS as seen in the entire evaluation, an approximately equal number of males $(7.0 \%, 15 / 213)$ and females $(6.6 \%, 14 / 213)$ were found to be infected with HIV. Among the age groups, the age group of $40-49$ years had the highest number $(6.1 \%, 13 / 213)$ of $\mathrm{HIV}$ infections; however, the distribution among the remaining age groups was statistically insignificant $\left(\chi^{2}=9.621, p=\right.$ 0.142 , likelihood ratio $=9.584, p=0.143$ ). The annual trend of HIV infection among clients on DOTS for the period is as indicated in Table $4\left(\chi^{2}=2.742, p=0.602\right.$, likelihood ratio $=$ 2.995, $p=0.559)$.

3.5. Treatment Outcome of TB/HIV Coinfected Clients. Pretreatment sputum smear grading among these HIV-infected clients showed that 14 out of the 29 (48.3\%) infected had no AFB. The remaining 15 were coinfected with TB. Of the 29 clients with TB and HIV coinfection, nine were cured, 13 completed the treatment, and 6 were not cured, while 1 was lost to follow-up (Figure 2). The fate of the one who was lost to follow-up could not be traced in the TB register. None of the clients with the HIV infection died during the period under review.

3.6. Analysis on TB/HIV Coinfection. Out of the 126 clients whose PSSG was positive (sc, $1+, 2+$, or $3+$ ), 15 were coinfected with HIV. This number represents $7.0 \%$ (15/213) of the total number of clients evaluated. The distribution of TB/HIV coinfection with regard to PSSG is as shown in Figure 3. With regard to their treatment outcome, 9 got cured and 5 died at home, while 1 had treatment failure (not cured). There was no default among the TB/HIV coinfected clients.

\section{Discussion}

A comprehensive evaluation of treatment outcomes is of supreme importance to the National TB Control Programme. Significant among strategic schemes to reduce the prevalence of TB infection worldwide is the annual evaluation 
TABLE 3: Pretreatment sputum smear grade and treatment outcome among clients.

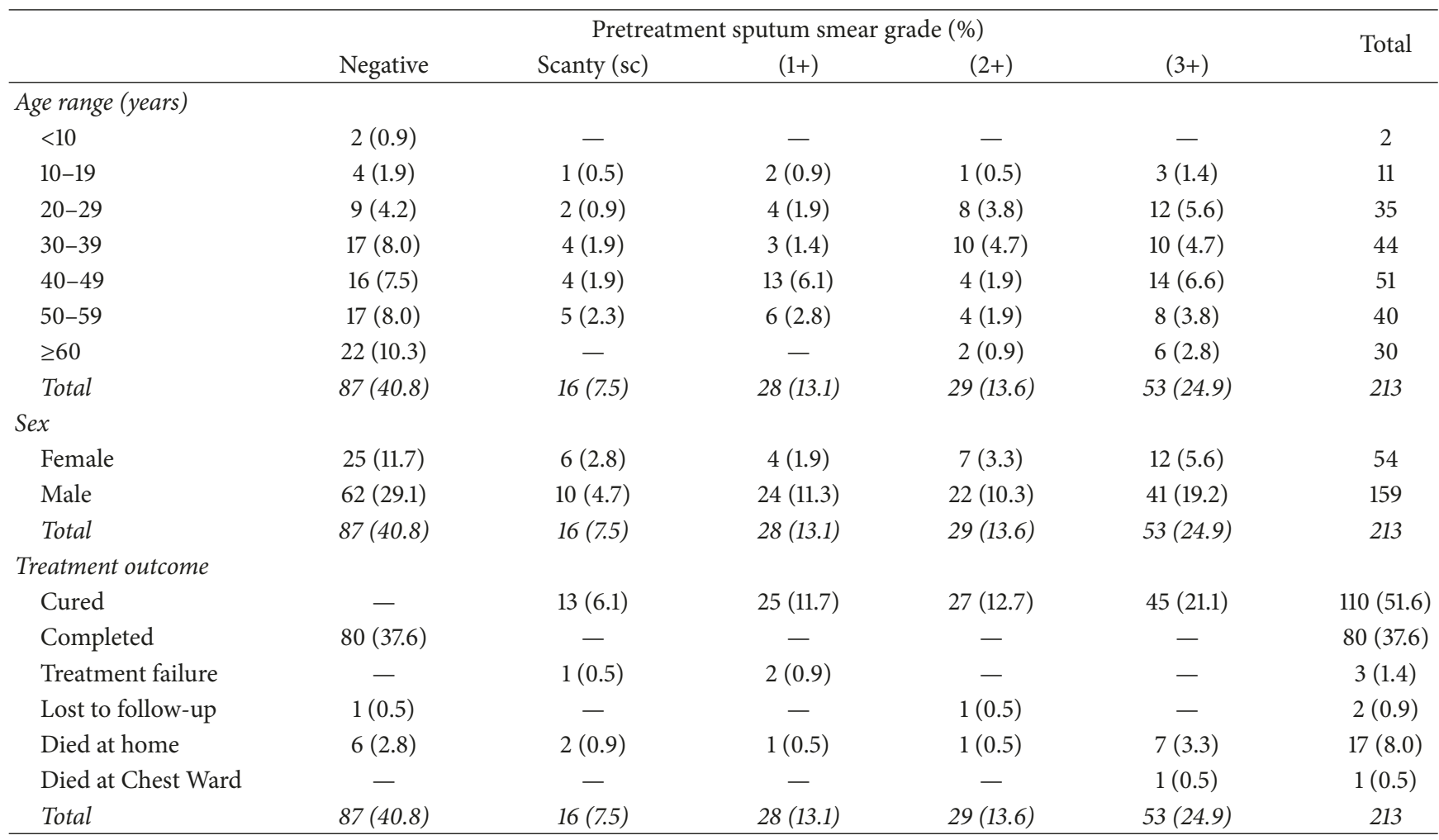

Negative: no Acid-Fast Bacilli (AFB) seen in at least 100 fields; scanty (sc): 1-9 AFB found in 100 fields; (1+): 10-99 AFB found in 100 fields; (2+): 1-10 AFB found per field in at least 50 fields; (3+): more than 10 AFB per field in at least 20 fields.

TABLE 4: HIV infection status among TB clients.

\begin{tabular}{lccc}
\hline \multicolumn{2}{c}{ HIV infection } & Total \\
\hline Sex & Positive (\%) & Negative & \\
Male & $15(7.0)$ & 144 & 159 \\
Female & $14(6.6)$ & 40 & 54 \\
Age & & & \\
$<10$ & - & 2 & 2 \\
$10-19$ & $1(0.5)$ & 10 & 11 \\
$20-29$ & $4(1.9)$ & 31 & 35 \\
$30-39$ & $6(2.8)$ & 38 & 44 \\
$40-49$ & $13(6.1)$ & 38 & 51 \\
$50-59$ & $2(0.9)$ & 38 & 40 \\
$\geq 60$ & $3(1.4)$ & 27 & 30 \\
Year & & & \\
2012 & $3(1.4)$ & 41 & 44 \\
2013 & $10(4.7)$ & 45 & 55 \\
2014 & $7(3.3)$ & 41 & 48 \\
2015 & $6(2.8)$ & 38 & 44 \\
2016 & $3(1.4)$ & 19 & 22 \\
\hline
\end{tabular}

of treatment outcomes in sputum smear-positive TB cases and among individuals with TB/HIV coinfection. This study sought to do that evaluation.

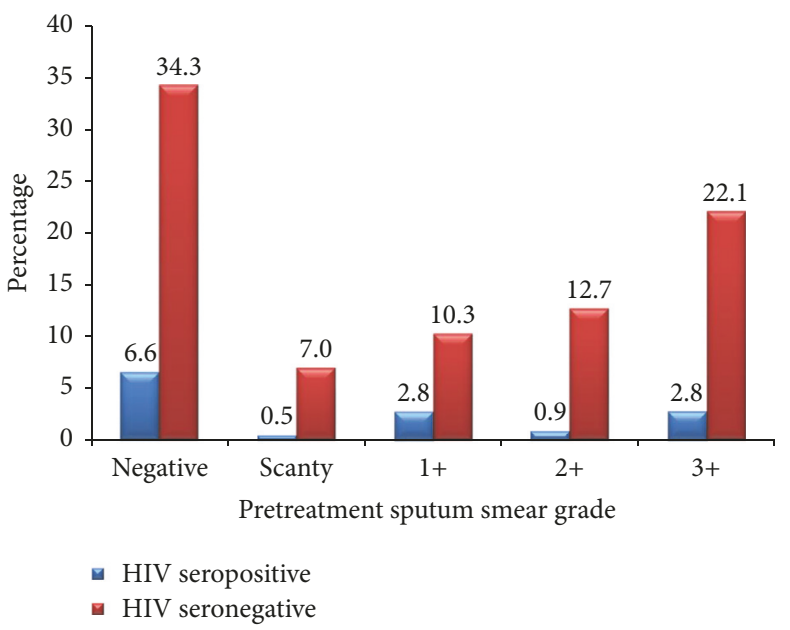

Figure 3: TB/HIV coinfection. Negative: no Acid-Fast Bacilli (AFB) seen in at least 100 fields; scanty (sc): 1-9 AFB found in 100 fields; (1+): 10-99 AFB found in 100 fields; (2+): 1-10 AFB found per field in at least 50 fields; (3+): more than 10 AFB per field in at least 20 fields.

Out of the total, $51.6 \%$ clients were "cured," while $37.6 \%$ completed their medication. In total, $90.2 \%$, which equals the WHO 2011-2015 updated cure rate target of $87.0 \%$, was achieved from 2012 to 2016 at the treatment site of the Metropolitan Hospital. This study's results suggest an improvement in treatment outcome. However, further 
studies are required to explain the factors associated with unfavourable outcomes, such as death and treatment failures at this TB treatment site as these could threaten the success of the National TB Control Programme in future.

Default rate throughout the entire period was $0.9 \%$ compared to the WHO interventional target of $5.0 \%$. This has been achieved through establishment of zonal treatment sites to maximize reach and supervision of adherence to medication. For example, all TB clients who accessed diagnostic services at our treatment site were referred back to treatment sites within their respective locations. Only clients within the Cape Coast Metropolitan Assembly were offered treatment, and this could have led to such an outcome. In addition, the treatment site adopts a facility based DOTS backed by a community strategy involving intensive defaulter tracing through house address and telephone number calling. These could have contributed to the increased adherence. The study, however, documented an $8.5 \%$ death rate in total. Death rate ranges from $7 \%$ to $35 \%$ worldwide [15-17]. Our results and interactions with nursing staff suggest that clients could have died at home due to lack of proper care and poor adherence to treatment regimen. Counselling, sustained supervision, home visits, and health education have been used successfully as interventions to reduce defaulter rate/loss to follow-up of TB patients [18].

This study showed that sex was not significantly associated with treatment outcome but two times more of the males died compared to females. Males are known to have poor health seeking behavior than females and usually seek medical attention late at advanced stages of the disease. Males are known to have poor adherence to treatment and often default treatment compared to females $[19,20]$.

Sputum smear-positive clients are infectious to close contacts and the general population throughout the treatment period. Following commencement of treatment, patients have a reduction in AFB load; however, they continue to expel viable bacilli for a variable period of time. In the present study, $8.4 \%$ [(sc: 9, 1+: 6, 2+: 1, and 3+: 2)/213] still had AFB after 2-3 months of treatment. At the end of the 5th month up till the 8th month, $1.9 \%$ [(sc: $1,1+: 2$, and $3+$ : 1)/213] remained not cured and would continue to expel viable bacilli. Treatment failure was seen in the age groups of $20-29(0.9 \%, 2 / 213)$ and $50-59(0.5 \%, 1 / 213)$ years. Two clients were lost to follow-up, one each in the age groups of 20-29 and $\geq 60$ years, respectively. Our findings are contrary to a study in Thailand, which showed that an age $\geq$ 60 years significantly correlated with treatment interruption and/or treatment failure [21]. Furthermore, the assertion in other studies [21-23] that an older age is a risk factor for death in pulmonary TB is incongruent with findings of this study. Out of 30 who died at home in this study, only one person was $\geq 60$ years old. Further studies are required in TB clients on DOTS to bring finality to inconsequential outcomes.

This study found $13.6 \%$ of clients on DOTS strategy within the period to be infected with HIV. Out of this, $6.1 \%$ was among the age group of $40-49$ years. Although males constituted the majority on DOTS, HIV prevalence of females, $6.6 \%$, almost equaled that of males, $7.0 \%$.
It is known that increased risk, as well as multidrugresistant (MDR) tuberculosis, is mostly found in clients who are HIV positive. In this study, two MDRs were identified: one was HIV positive, while the other was HIV negative. Although studies in Ethiopia have shown high default likelihood among TB/HIV coinfected clients $[19,20]$, our study did not show any default among this category. In total, the number of clients with TB/HIV coinfection was 15 (7.0\%). TB/HIV coinfection was highest (7/15) among the age group of 40-49 years. The potential of this age group being economically and sexually active cannot be substantiated without further studies. Since the overall number of HIV positive patients in the study is small, we could not establish the impact of HIV on sputum smear conversion. Literature search is yet to establish any effect in clients with $\mathrm{TB} / \mathrm{HIV}$ coinfection $[9,24,25]$. Overall, the DOTS strategy has performed well, requiring continuous modifications and reviews to enhance its outcome.

\section{Conclusion}

We found a high treatment success rate among clients treated at our site. The relevant strategies to ensure early detection and treatment are under implementation. As expected, administration of medication on the wards is supervised by trained staff. Provision has also been made for trained staff to administer the DOTS and SLDs in communities where clients live. The information currently lacking is the potential negative sociodemographic/economic factors that result in unfavourable outcomes not controlled by the DOTS strategy.

\section{Conflicts of Interest}

The authors declare that they have no conflicts of interest.

\section{Authors' Contributions}

Ato Kwamena Tetteh and Edward Agyarko conceived and designed the study. They both participated in data analysis and the drafting of the manuscript. Irene Ayi, Langbong Bimi, and Joseph Otchere provided technical advice and contributed immensely to the writing of the manuscript.

\section{Acknowledgments}

The authors would like to thank the Officer in Charge of the Chest Centre and supporting Staff Nurses for cooperating with them during data collation and entry. They acknowledge their unrelenting patience in explaining all technical definitions regarding tuberculosis management at the facility. The authors' profound gratitude also goes to Dr. Charles Gyamfi, Department of Civil Engineering, Tshwane University of Technology, Pretoria, South Africa, for providing them with the map of Cape Coast Metropolitan Area.

\section{References}

[1] W. Wobeser, L. Yuan, and M. Naus, “The Tuberculosis Treatment Completion Study Group. Outcome of pulmonary tuberculosis treatment in the tertiary care setting-Toronto 1992/93," 
Canadian Medical Association Journal, vol. 160, pp. 789-794, 1999.

[2] Global tuberculosis Control, Surveillance, Planning, FinancingWHO Report 2005: WHO/HTM/TB/2005, vol. 49, WHO, Geneva, Switzerland, 2005.

[3] E. Owusu-Dabo, O. Adjei, C. G. Meyer et al., "Mycobacterium tuberculosis Drug Resistance, Ghana. Letter to the Editor," Emerging Infectious Diseases, vol. 12, no. 7, pp. 1170-1172, 2006.

[4] A. Trebueq and H. L. Reider, "Two excellent management tools for national tuberculosis programmes; history of prior treatment and sputum status at two months," International Journal of Tuberculosis and Lung Disease, vol. 2, pp. 184-186, 1998.

[5] F. Torman, Ed., Tomans tuberculosis case detection, treatment, and monitoring-questions and answers, World Health Organisation, Geneva, Switzerland, 2nd edition, 2004.

[6] TB. Global Report, 2011.

[7] S. P. Agarwal and L. S. Chauhan, "Tuberculosis control in India," in Directorate general of Health services, Ministry of Health and Family Welfare, government of India, New Delhi, India, 2005.

[8] R. Long, K. Bochar, S. Chomyc et al., "Relative Versus Absolute Noncontagiousness of Respiratory Tuberculosis on Treatment," Infection Control and Hospital Epidemiology, vol. 24, no. 11, pp. 831-838, 2003.

[9] E. E. Telzak, B. A. Fazal, C. L. Pollard, G. S. Turett, J. E. Justman, and S. Blum, "Factors influencing time to sputum conversion among patients with smear- positive pulmonary tuberculosis," Clinical Infectious Diseases, vol. 25, no. 3, pp. 666-672, 1997.

[10] M. S. Al-Moamary, W. Black, E. Bessuille, R. K. Elwood, and S. Vedal, "The significance of the persistent presence of acid-fast bacilli in sputum smears in pulmonary tuberculosis," CHEST, vol. 116, no. 3, pp. 726-731, 1999.

[11] P. G. Gopi, V. Chandrasekaran, R. Subramani et al., "Association of conversion \& cure with initial smear grading among new smear positive pulmonary tuberculosis patients treated with Category I regimen," Indian Journal of Medical Research, vol. 123, no. 6, pp. 807-814, 2006.

[12] R. Singla, M. M. Osman, N. Khan, N. Al-Sharif, M. O. AlSayegh, and M. A. Shaikh, "Factors predicting persistent sputum smear positivity among pulmonary tuberculosis patients 2 months after treatment," International Journal of Tuberculosis and Lung Disease, vol. 7, pp. 58-64, 2003.

[13] J.-Y. Wang, L.-N. Lee, C.-J. Yu, Y.-J. Chien, and P.-C. Yang, "Factors influencing time to smear conversion in patients with smear-positive pulmonary tuberculosis," Respirology, vol. 14, no. 7, pp. 1012-1019, 2009.

[14] T. H. Holtz, M. Sternberg, S. Kammerer et al., "Time to sputum culture conversion in multidrug-resistant tuberculosis: Predictors and relationship to treatment outcome," Annals of Internal Medicine, vol. 144, no. 9, pp. 650-659, 2006.

[15] C. Dye, S. Scheele, P. Dolin, V. Pathania, and M. C. Raviglione, "Global burden of tuberculosis: estimated incidence, prevalence, and mortality by country. WHO Global Surveillance and Monitoring Project," Journal of the American Medical Association, vol. 282, no. 7, pp. 677-686, 1999.

[16] P. J. Dolin, M. C. Ranglione, and A. Kochi, "Global tuberculosis incidence and mortality during," Bulletin of the World Health Organization, vol. 72, Article ID 19902000, pp. 213-20, 1994.

[17] M. C. Ranglione, D. E. Snider Jr., and A. Kochi, "Global epidemiology of tuberculosis: morbidity and mortality of a worldwide epidemic," Journal of the American Medical Association, vol. 273, no. 3, pp. 220-226, 1995.
[18] G. Berhe, F. Enquselassie, and A. Aseffa, "Treatment outcome of smear-positive pulmonary tuberculosis patients in Tigray Region, Northern Ethiopia," BMC Public Health, vol. 12, no. 1, article 537, 2012.

[19] G. Mitike, D. Kebede, and H. Yeneneh, "HIV infection and antituberculosis drug resistance among pulmonary tuberculosis patients in Harar tuberculosis centre, Ethiopia," East African Medical Journal, vol. 74, no. 3, pp. 154-157, 1997.

[20] S. Vijay, P. Kumar, L. S. Chauhan, S. V. Rao, and P. Vaidyanathan, "Treatment outcome and mortality at one and half year followup of HIV infected TB patients under TB control programme in a district of South India," PLoS ONE, vol. 6, no. 7, Article ID e21008, 2011.

[21] S. Anunnatsiri, P. Chetchotisakd, and C. Wanke, "Factors associated with treatment outcomes in pulmonary tuberculosis in northeastern Thailand," Southeast Asian Journal of Tropical Medicine and Public Health, vol. 36, no. 2, pp. 324-330, 2005.

[22] K. C. Takarinda, A. D. Harries, S. Srinath, T. Mutasa-Apollo, C. Sandy, and O. Mugurungi, "Treatment outcomes of adult patients with recurrent tuberculosis in relation to HIV status in Zimbabwe: A retrospective record review," BMC Public Health, vol. 12, no. 1, article no. 124, 2012.

[23] M. A. Yassin, L. Takele, S. Gebresenbet et al., "HIV and tuberculosis coinfection in the southern region of Ethiopia: a prospective epidemiological study," Infectious Diseases, vol. 36, no. 9, pp. 670-673, 2004.

[24] A. Dominguez-Castellano, M. A. Muniain, J. Rodriguez-Bano et al., "Factors associated with time to sputum smear conversion in active pulmonary tuberculosis," Int J Tuberc Lung Dis, vol. vol 7, pp. 432-438, 2003.

[25] R. Bwire, M. W. Borgdorff, V. Sticht-Groh et al., "Tuberculosis chemotherapy and sputum conversion among HIV seropositive and HIV sero-negative patients in south-earthern uganda," East African Medical Journal, vol. 76, pp. 307-313, 1999. 


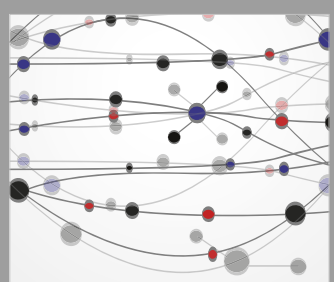

The Scientific World Journal
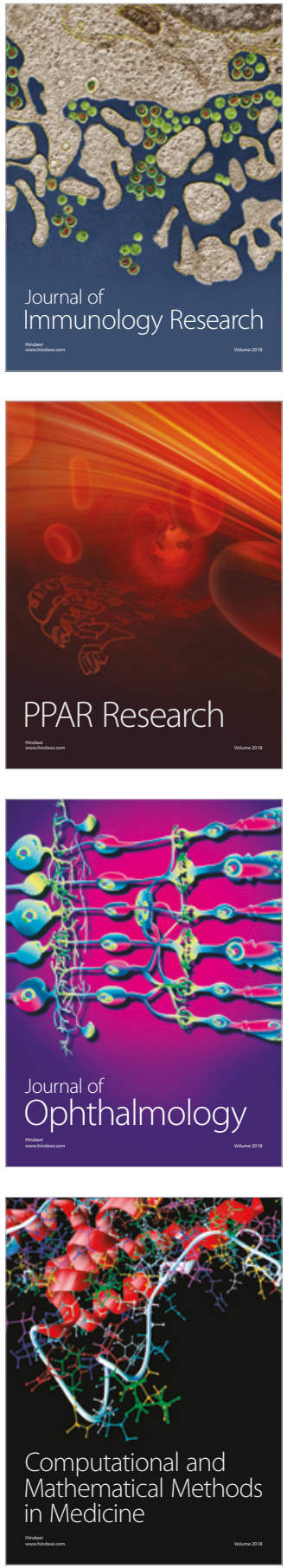

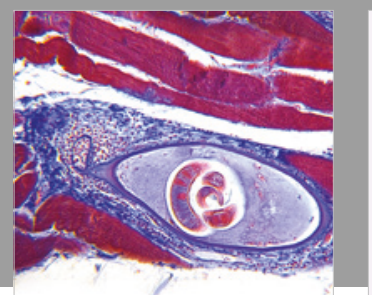

Gastroenterology Research and Practice

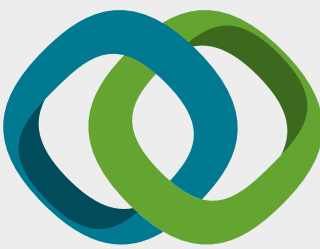

\section{Hindawi}

Submit your manuscripts at

www.hindawi.com
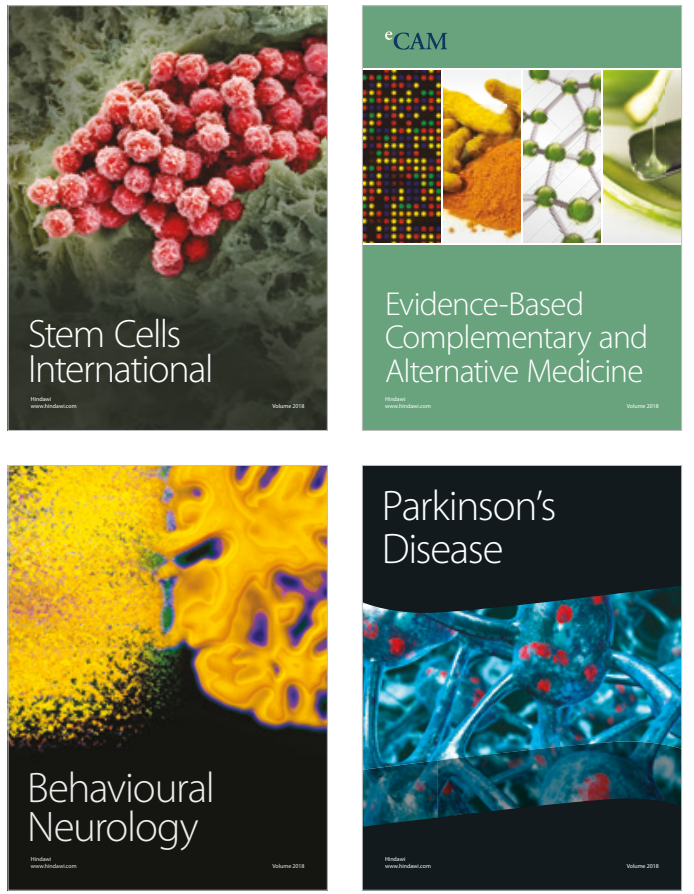

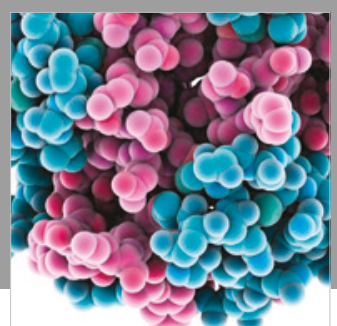

ournal of

Diabetes Research

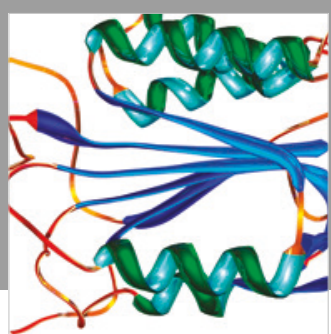

Disease Markers
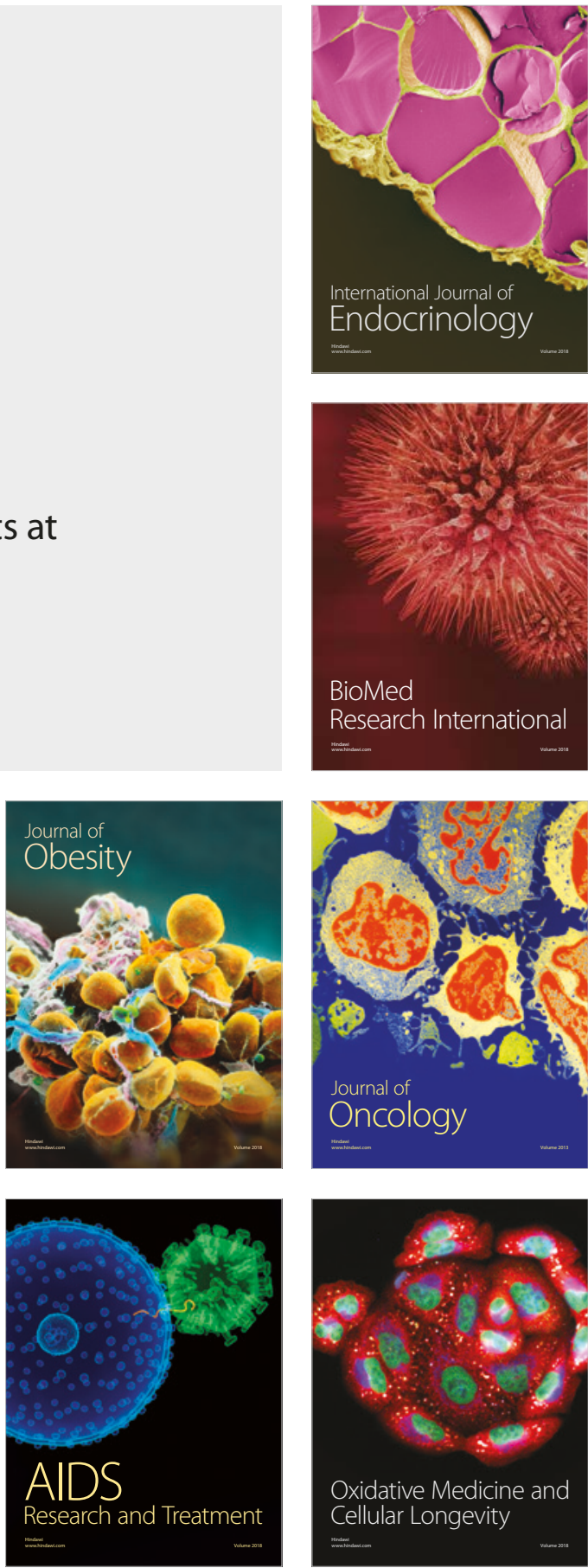